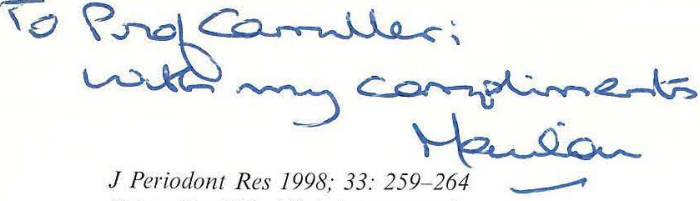

Printed in UK. All rights reserved
Pamphlet

- Box ..
Copyright (O Munksgaard 1998

JOURNAL OF

PERIODONTAL RESEARCH

ISSN 0022-3484

\section{Periotron 8000: calibration characteristics and reliability}

Ciantar M, Caruana DJ: Periotron 8000: calibration characteristics and reliability. J Periodont Res 1998; 33: 259-264. (C) Munksgaard, 1998.

The Periotron is an instrument designed to quantify submicrolitre volumes of fluid sampled on a filter paper strip. To date 3 models have been manufactured: the Periotron 600 (1976), the Periotron 6000 (1983) and more recently the Periotron 8000 (1995). This paper investigated for the first time the calibration characteristics and reliability of the Periotron 8000 . The fluids under investigation were: de-ionised water, human serum, fetal bovine serum and an ultrafiltrate of fetal bovine serum. Quantitative analysis was studied by recording a series of Periotron readings over a volume range of $0-1.0 \mu \mathrm{l}$ for each fluid. The average of 5 Periotron values for each particular fluid was then plotted versus the respective fluid volume. Qualitative changes in fluid composition versus Periotron Scores were also analysed. Volume conversion for Periotron scores using both Periotron MLCONVRT software and a best fit equation selected from TableCurve 2D software compared well. The results of this study revealed that: 1) differences in calibration fluid composition (e.g. protein content) are reflected in the Periotron scores; 2) positioning of filter paper strip between the jaws of the Periotron should be standardised, 3) calibration of the Periotron 8000 seems to be consistent over a 1 -wk interval.

\section{Ciantar ${ }^{1}$, D. J. Caruana ${ }^{2}$}

${ }^{1}$ Department of Dental Surgery, University of Malta, Msida, Malta, ${ }^{2}$ Department of Biomedical Sciences, University of Malta, Msida, Malta
M. Ciantar, Department of Dental Surgery, University of Malta, Msida, Malta

Key words: Periotron; calibration; submicrolitre volumes; gingival crevicular fluid

Accepted for publication March 10, 1998
The potential role of gingival crevicular fluid (GCF) as aid in periodontal diagnosis is well documented (1-4). One of the major goals of GCF research is to devise techniques capable of detecting quantitative/qualitative changes predictive of periodontal pathology. GCF originates from tissue fluid which percolates through the junctional epithelium into the gingival crevice/periodontal pocket. Hence analysis of its biochemical components reflects the situation within the tissues and crevice.

Biochemical analyses of GCF are currently receiving a great deal of attention. Studies involving GCF have yielded promising results, and to date some 40 GCF components have been studied (5-10). Measurements of GCF constituents can be expressed either in terms of molar concentration, units of activity per unit volume, or total amount of sample per unit time. Methods employed for collecting GCF are: i) pre-cut filter paper strips placed at the orifice, partly into or inserted to the bottom of the pocket or sulcus, ii) paper points and capillary tubes inserted subgingivally, and iii) gingival washings (4). Volumetric quantitation of GCF may be estimated either by using microcapillary tubes of known internal diameter, or an electronic device, e.g. Periotron 8000.

The Periotron 8000 (Pro-Flow Inc., Amityville, New York, USA) quantifies the volume of gingival fluid/saliva collected on filter paper. It functions on the principle of the capacitor, i.e. it measures the electrical capacitance of a wet filter paper strip placed between the jaws of the instrument. The electric field created by opposing charges on the jaws induces polarity of the molecules which reduces the potential difference between the plates and increases the capacitance. Thus the higher the number of polar molecules between the plates (jaws) of the Periotron, the larger the capacitance and the higher the Periotron scores. Unknown fluid volumes on filter paper strips may be determined from calibration graphs constructed by using accurately measured quantities of fluid.

The Periotron 8000 is a new model (1995). It adds computer capability to its previous version, the Periotron 6000. To date the periodontal literature does not include studies pertaining to the 8000 model. The aim of this study was to investigate the 
calibration characteristics and reliability of the Periotron 8000.

\section{Materials and methods}

The experiment protocol was based on that described by Preshaw et al. (11); however, some modifications were made: fluid volumes $(0-1.0 \mu \mathrm{l})$ at $0.05 \mu \mathrm{l}$ increments were dispensed with a P2 Gilson microsyringe (Gilson, 72 rue Gambetta, BP 45, 95400, Villiers-le-Bel, France) onto Periopaper strips (Pro-flow Inc., Amityville, New York, USA). The fluid was applied to the edge of the filter paper strip held in an upright position allowing downward fluid spread along the strip. Each strip was immediately placed between the jaws up to a depth of $0.5 \mathrm{~mm}$ from the orange handle. Exact positioning of different strips was achieved by securing a plastic sleeve to the lower jaw of the Periotron. The plastic sleeve had a mid-line horizontal slot level with the lower jaw and of sufficient width to just allow entrance of the filter paper strip. The reading on the LED display at the end of the 16-s cycle was automatically recorded by the Periotron computer software program. A series of Periotron scores was recorded for the range of volumes. The procedure was repeated 5 times and the average Periotron score for each volume calculated.

The fluids under investigation were de-ionised water (W), human serum (HS), fetal bovine serum (Gibco BRL, Life Technologies Ltd, Paisley, Scotland) (FBS) and centrifuged fetal bovine serum or ultrafiltrate (UFBS). Human serum was derived from a blood sample obtained by venepuncture, allowed to clot, then centrifuged at $1000 \mathrm{rpm}$ for $20 \mathrm{~min}$. A sample of fetal bovine serum was centrifuged at $6500 \mathrm{rpm}$ for $50 \mathrm{~min}$ in a microcon tube (Amicon Inc., Beverly, USA) having a membrane cut-off point of $3 \mathrm{kdal}$. The UFBS was used to identify and assess any changes in the Periotron scores following qualitative changes (removal of proteins) in the serum after centrifugation.

A second set of readings for de-ionised water was obtained during a second calibration experiment performed one week after the first set of results.

The protein content of the fluids under investigation was determined using a modified version of the Bradford protein assay (Bio-Rad Protein Assay, Bio-Rad Laboratories $\mathrm{GmbH}$, Heidemannstraße 164, D-80939, München, Germany).

\section{Results}

The average of 5 Periotron scores were plotted versus the corresponding volume of fluid $(\mu \mathrm{l})$ for $\mathrm{W}, \mathrm{HS}, \mathrm{FBS}$ and the UFBS. The individual data sets were analysed using TableCurve 2D curve fitting software supplied by Jandel (Jandel GmbH, Schummelstrasße 25, 40699, Erkrath, Germany). The software fits over 8000 pre-programmed candidate equations to the data using either GaussJordan or Singular value decomposition fitting procedures. Effectively, all the points on the calibration curve are relative to a zero point, or a filter paper strip at ambient conditions with no applied fluid. Therefore, equations which do not pass through zero were eliminated. Equations with more than 4 coefficients were also eliminated because the curve fitting was not stable and errors in the coefficients were significant. As a result only simple equations with 4 coefficients or less which describe a function through zero were selected. A summary of the 5 best fitting equations identified by TableCurve 2D are shown in Table 1.

With the exception of water, the equation

$y=a+b x^{\mathrm{c}}$

where $y$ represents the Periotron score, $a$ the intercept $(=0), x$ the fluid volume and $b, c$ and $d$ are function coefficients unique for every fit, fitted most suitably as shown by the high regression coefficient $\left(r^{2}\right)$ and low fit standard error (FSE).

The 5 equations were applied to the Periotron results generated using human serum. Graphic presentations (Periotron scores versus volume of serum in $\mu \mathrm{l}$ ) shown in Fig. 1, demonstrate that Eqn 1 gives the best fit.

The graphs resulting from calibration experiments using W over a volume range of $0-1.0 \mu \mathrm{l}$ performed 1 wk apart are presented in Fig. 2. The similarity between the 2 plots can be accounted for by the comparable values of the coefficients $b$ and $c$ in Eqn 1 (legend to Fig. 2). The reliability of the Periotron 8000 for replicate experiments on different days was very good, but frequent calibration is still recommended in clinical studies.

The Periotron scores for all 4 fluids are compared in Fig. 3. The protein content of the different sera was estimated to be $(\mathrm{mg} / \mathrm{ml})$ : UFBS $=5.09$, $\mathrm{FBS}=30.87$ and $\mathrm{HS}=60.24$. At a specified volume of fluid, Periotron scores for the UFBS were higher than those for FBS and the latter in turn higher than those for HS. These results demonstrated that the protein content of the fluid affects Periotron readings. These 3 curves show that, the higher the protein content of the fluid under investigation, the lower the Periotron score. This is also reflected in Table 2 where the volumes are calculated from Periotron readings using the first equation in Table 1. The curve for water lay between that for the UFBS and FBS. The same result was also noted in Table 2. 
Table 1. Summary of 5 best-fitting equations applied for W, HS, FBS and UFBS as determined by TableCurve; $r^{2}=$ regression coefficient; FSE = fit standard error: the least squares error of fit; the closer the value is to zero the better the fit; $a$, the intercept $=$ 0 in all equations

\begin{tabular}{|c|c|c|c|c|c|}
\hline Equation & & W & HS & FBS & UFBS \\
\hline$y=a+b x^{\mathfrak{c}}$ & $\begin{array}{l}r^{2} \\
\text { FSE } \\
\mathrm{b} \\
\mathrm{c}\end{array}$ & $\begin{array}{c}0.9979 \\
2.0176 \\
153.3 \\
0.771\end{array}$ & $\begin{array}{c}0.995 \\
2.558 \\
135.0 \\
0.834\end{array}$ & $\begin{array}{c}0.9961 \\
2.5835 \\
149.3 \\
0.790\end{array}$ & $\begin{array}{c}0.9972 \\
2.2557 \\
156.4 \\
0.704\end{array}$ \\
\hline$y^{2}=a+b x^{1.5}$ & $\begin{array}{l}r^{2} \\
\text { FSE } \\
\mathrm{b}\end{array}$ & $\begin{array}{r}0.9974 \\
2.149 \\
23,121.7\end{array}$ & $\begin{array}{c}0.9901 \\
3.77 \\
17,053.6\end{array}$ & $\begin{array}{c}0.9947 \\
2.95 \\
21,595.3\end{array}$ & $\begin{array}{r}0.995 \\
2.948 \\
25,267.1\end{array}$ \\
\hline$y=a+b x$ & $\begin{array}{l}r^{2} \\
\text { FSE } \\
b\end{array}$ & $\begin{array}{r}0.958 \\
8.694 \\
165.1\end{array}$ & $\begin{array}{c}0.9776 \\
5.664 \\
142.4\end{array}$ & $\begin{array}{r}0.964 \\
7.653 \\
159.8\end{array}$ & $\begin{array}{r}0.919 \\
11.878 \\
172.2\end{array}$ \\
\hline$y=a+b x+c x^{2}+d x^{3}$ & $\begin{array}{l}r^{2} \\
\text { FSE } \\
b \\
c \\
d\end{array}$ & $\begin{array}{c}0.9988 \\
1.5212 \\
248.5 \\
-169.1 \\
73.85\end{array}$ & $\begin{array}{c}0.9958 \\
2.5741 \\
203.6 \\
142.0 \\
76.1\end{array}$ & $\begin{array}{c}0.99516 \\
2.999 \\
249.8 \\
-216.0 \\
120.6\end{array}$ & $\begin{aligned} & 0.9968 \\
& 2.4912 \\
& 290.0 \\
&-245.4 \\
& 112.3\end{aligned}$ \\
\hline$y=a+b x+c x^{2}$ & $\begin{array}{l}r^{2} \\
\text { FSE } \\
\mathrm{b} \\
\mathrm{c}\end{array}$ & $\begin{array}{c}0.9964 \\
2.586 \\
217.6 \\
-68.3\end{array}$ & $\begin{array}{c}0.9926 \\
3.323 \\
171.7 \\
-38.2\end{array}$ & $\begin{array}{c}0.988 \\
4.529 \\
199.3 \\
-51.4\end{array}$ & $\begin{array}{r}0.9912 \\
4.0356 \\
243.0 \\
-92.1\end{array}$ \\
\hline
\end{tabular}

\section{Discussion}

Factors known to affect readings of previous models, the Periotron 600 and 6000 , were identified and taken into consideration before performing this study. Variables which were kept constant throughout the trial were: fluid constituents under investigation (12-14), strip location between the jaws $(13,15)$, quality and dimensions of filter paper strip $(16,17)$ and ambient humidity and temperature (15). Strip positioning, both relative to midline as well as depth of insertion of filter paper strips between the jaws, affects Periotron readings $(13,15)$. Strip placement was standardised by using a plastic sleeve affixed to the lower jaw.

The quality and dimensions of the filter paper strips are known to affect the amount of fluid absorbed (16-18). Previous studies $(4,17,18)$ have shown that fluid flow measurements using different types/dimensions of filter paper strips should not be compared. Thus filter paper strip standardization was ensured by using those supplied with the Periotron. Fluids were applied at the upper end of filter paper strips and allowed to spread downwards to avoid discrepancies in the rate of fluid flow between ascending and descending fluid movements (18).

Environmental factors affect the rate of fluid evaporation from the filter paper strips (15); however, during this trial the laboratory conditions were relatively constant with average ambient temperature $=20^{\circ} \mathrm{C}$ and humidity $=40 \%$.

The plot of Periotron scores against fluid volume for the Periotron 600 and 6000 have been described in a number of ways. The calibration curves do not seem to follow a simple physiochemical behaviour, and as a result have been interpreted as being linear (14), curvilinear (19), quadratic $(11,13,17)$, as having 2 different linear regressions (20), or also as a 3-section graph with two linear sections and a curve (21). The purpose of graph characterization using a mathematical function is to determine the liquid volume of a GCF sample from a Periotron score by using an equation which best describes the data in the calibration curve.

Eqn 1 provided a good method of calculating the volume from the Periotron scores obtained. Although the difference between volumes estimated using the 5 equations were small, statistically Eqn 1 fitted the data more closely.

The Periotron 8000 has a computer software program designed to automatically set up a database of known Periotron fluid measurements. A calibration graph over a volume range of $0-0.7 \mu \mathrm{l}$ at $0.1 \mu \mathrm{l}$ increments plotted using values from this database may subsequently be used to quantify unknown volumes of fluid applied on a filter paper strip. The Periotron scores are then converted into microlitres by using the MLCONVRT program.

In this investigation the Periotron scores were recorded using the software supplied with the Periotron 8000 . Conversion of the Periotron scores into the corresponding volumes in $\mu l$ was calculated using both the MLCONVRT software and Eqn 1 (Table 3). In gencral the volumes obtained for the Periotron scores were comparable using both 


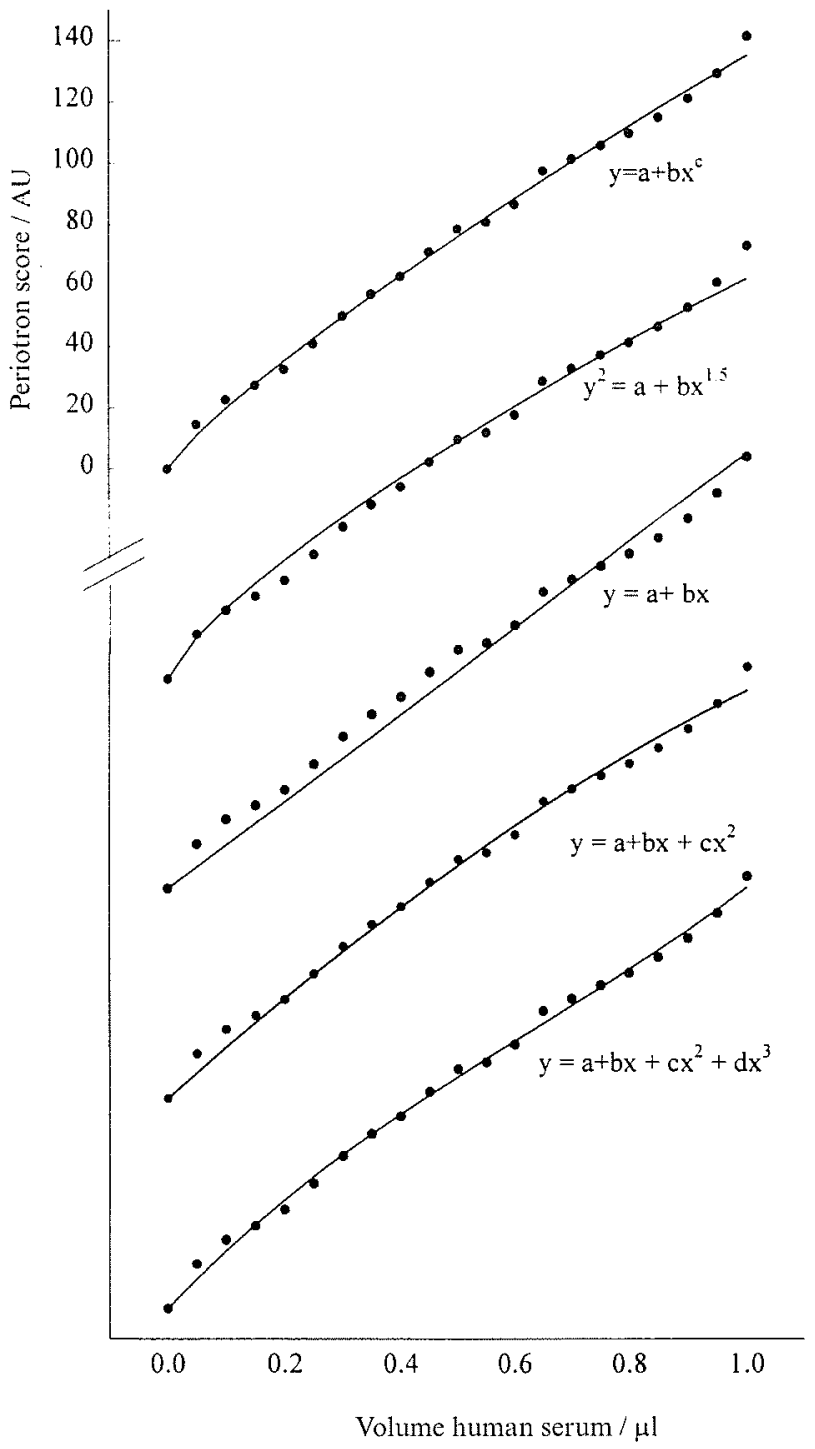

Fig. 1. Plots of Periotron score versus volume $(\mu 1)$ of human serum with the best fit of the equations (solid line) shown on the graph and Table 1.

methods. Extrapolation of volumes above $0.7 \mu \mathrm{l}$ is not reliable using polynomials. The Periotron program fits a fourth order polynomial equation to the data, although the details of the curve-fitting process are not described in the accompanying information. From the analysis of the data obtained from TableCurve 2D using conventional algorithms to fit polynomial and rational equations, the coefficients tended to be very large and alternating in sign. The equations fitted well to the data but were not stable - the errors in the coefficients were frequently larger than the coefficients themselves. By far the greatest advantage of using the MLCONVRT software is that it provides a standardized method by which the Periotron scores may be converted to volumes. This will eliminate discrepancies of volume conversion using different

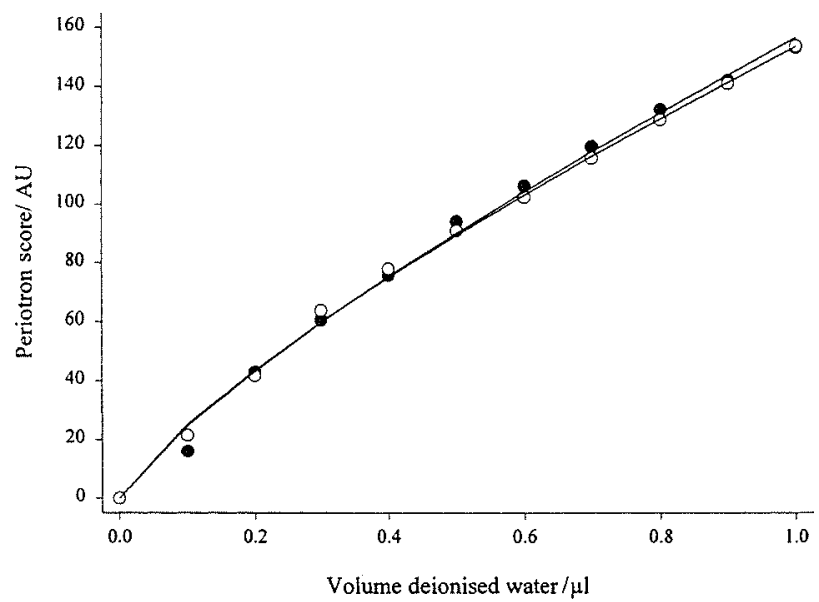

Fig. 2. Plots of Periotron score versus volume $(\mu \mathrm{l})$ of deionised water recorded 1 wk apart. Coefficients for the best fit to Eqn 1 are $b=156.4, c=0.80$ and $b=153.4, c=0.78$.

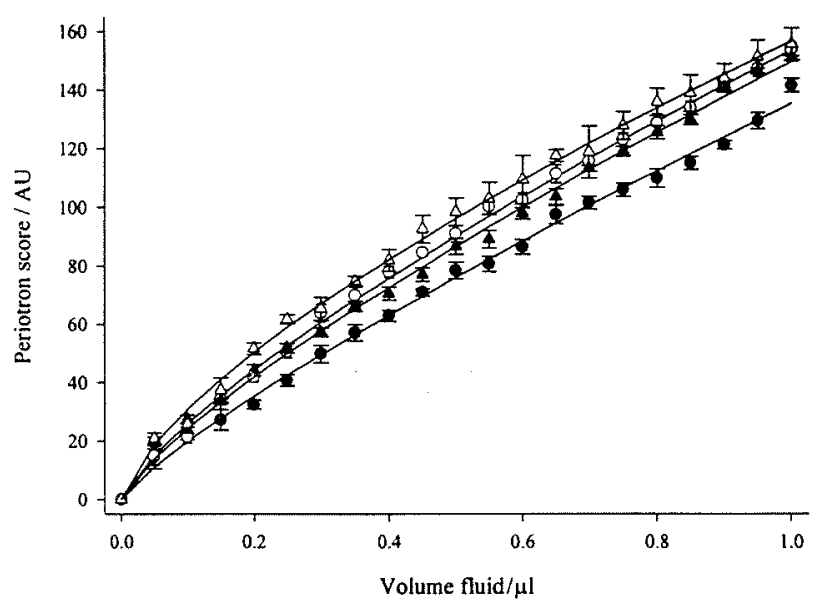

Fig. 3. Plots of Periotron score versus volume of UFBS (open triangle), water (open circles), FBS (filled triangles) and human serum (filled circles) in $\mu 1$. The solid lines are the best fit lines described by Eqn 1 with coefficients given in Table 1 .

Table 2. The volume calculated from Eqn 1 fitted to the respective calibration fluid

\begin{tabular}{lllll}
\hline $\begin{array}{l}\text { Periotron } \\
\text { units }\end{array}$ & W & HS & BS & UFBS \\
\hline 20 & 0.0713 & 0.101 & 0.0897 & 0.054 \\
60 & 0.296 & 0.378 & 0.3349 & 0.2567 \\
100 & 0.574 & 0.697 & 0.618 & 0.53 \\
130 & 0.8069 & 0.955 & 0.846 & 0.769 \\
\hline
\end{tabular}

methods of describing the calibration curves, as was reported for the Periotron 6000.

Due to the small amount of GCF which exudes through the gingival crevice it is unlikely to collect sufficient quantities to calibrate the Periotron (19, 21). Human serum was used as the calibrating fluid as it is the fluid which most closely simulates GCF in composition $(4,19,21,22)$. Other sera/fluids yielded different results (Table 2 ). 
Table 3. Comparison of the Periotron units to volume conversion using the periotron MLCONVRT program supplied with the Periotron and Eqn 1 with the coefficients from Table 1 for human serum and water

\begin{tabular}{rlcll}
\hline & $\begin{array}{c}\text { Periotron } \\
\text { (HS) }\end{array}$ & $\begin{array}{c}\text { Periotron } \\
\text { (water) }\end{array}$ & $\begin{array}{c}\text { Eqn 1 } \\
\text { (HS) }\end{array}$ & $\begin{array}{c}\text { Eqn 1 } \\
\text { (water) }\end{array}$ \\
\hline 20 & 0.10 & 0.10 & 0.101 & 0.0713 \\
60 & 0.38 & 0.29 & 0.378 & 0.296 \\
100 & 0.7 & 0.58 & 0.697 & 0.574 \\
130 & 0.0 & 0.0 & 0.955 & 0.806 \\
\hline
\end{tabular}

The calibration experiment with water was run twice, 1 week apart. The resulting calibration graphs (Fig. 2) were very similar, showing that for the same fluid and under the same ambient conditions, the Periotron 8000 yields consistent results.

The calibration graphs for UFBS, FBS, HS and $\mathrm{W}$ are compared in Fig. 3. Periotron scores for HS were less than those for $\mathrm{W}$. These results agree with those of Van der Bijl et al. (13) and Chapple et al. (21).

The 3 sera were compared to investigate the effect of different protein concentrations in the fluids on Periotron scores. The results show that as the protein content of the fluid increased, lower Periotron scores were recorded. The higher protein content caused slower spreading of the serum, a smaller wetted filter paper area and hence lower Periotron scores. In this regard the findings of this study agree with those of Leirskar (18) who demonstrated that the mobility of protein solutions on filter paper was inversely related to protein concentration. However, this may not be the only factor which contributed to the different scores. Water has a very high dielectric constant (78.2) (23) compared to that of proteins and ions. As a consequence the Periotron score is predominantly dependent on the quantity of water present in the filter paper strip. The curve for water lay between that for UFBS and FBS. Since water contains no proteins, we would have expected this to lie above that for UFBS. The exact reason for this is not known but we may speculate that the different scores obtained for the different fluids may not only be a direct result of the content of the fluid but also due to some other physiochemical effect, perhaps adsorption of ions and/or proteins on the electrode surface. As a consequence, direct comparison between widely differing solutions such as sera and water should not be performed. The difference between scores for the different fluids is small, but it is large enough to be significant in the context of this study.

More studies on the protein content of GCF need to be done to assess whether this protein factor is significant when using the Periotron 8000 for clinical studies. The fluid used for calibrating the Periotron 8000 should be similar in protein content to GCF. Human serum is one such fluid $(23-25)$. Further investigations are required into the fluid factors which affect Periotron scores.

\section{Acknowledgements}

This study was kindly supported by the Faculty of Dental Surgery, University of Malta. The authors wish to thank Prof. J. M. Portelli, Dean and Head of Department of Dental Surgery, and Prof. W. H. Bannister, Head of Department of Biomedical Sciences for their support and Dr H.-H. Frey for his contribution.

\section{References}

1. Black GV. Phagedena Pericementi. Illinois State Dental Soc 1882; 93-114.

2. Lamster IB. Evaluation of components of gingival crevicular fluid as diagnostic tests. Ann Periodontol 1997; 2: 123-127.

3. Page C. Host response tests for diagnosing periodontal diseases. $J$ Periodontol 1992; 63: 356-366.

4. Cimasoni GO. The crevicular fluid. In: Myers HM, ed. Monographs in oral science, vol. 3. Basel: Karger, 1974.

5. Lamster IB, Hartley LJ, Vogel RI. Development of a biochemical profile for gingival crevicular fluid. $J$ Periodontol 1985; 56 (suppl): 13-21.

6. Chapple ILC, Glenwright HD, Matthews JB, Thorpe GHG, Lumley PJ. Site specific alkaline phosphatase levels in gingival crevicular fluid in health and gingivitis: crosssectional studies. J Clin Periodontol 1994; 21: 409-414.

7. Adonoganiaki E, Mooney J, Kinane D. The ability of gingival crevicular fluid acute phase proteins to distinguish healthy, gingivitis and periodontitis sites. J Clin Periodontol 1992; 19: $98-102$.

8. Ciancio SG, Golub LM, Mosovich L, Katz C, Kleinberg I. Urea levels in the gingival crevices of diabetic and normal adolescents. J Dent Res 1977; 56: 1144.

9. Hara K, Loe H. Carbohydrate components of the gingival exudate. J Periodont Res 1969; 4: 202-207.

10. Hou LT, Liu CM, Rossomando EF. Crevicular interleukin-1B in moderate and severe periodontitis patients and the effect of phase 1 periodontal treatment. $J$ Clin Periodontol 1995; 22: 162-167.

11. Preshaw PM, Kelly PJ, Heasman PA. Quadratic calibration curves for the Periotron 6000. J Periodont Res 1996; 31: 441-443.

12. Garnick JJ, Person R, Harrell D. The evaluation of the Periotron. J Periodontol 1979; 50: 424-426.

13. Van der Bijl, Dreyer WP, Grobler SR. The Periotron gingival crevicular fluid meter. $J$ Periodont Res 1986; 21: 39-44.

14. Bickel M, Cimasoni G. Reliability of volume measurements with the new Periotron 6000. J Periodont Res 1984; 19: 313-316

15. Suppipat N, Suppipat N. Evaluation of an electronic device for gingival fluid quantitation. J Periodontol 1977; 48: 388-394.

16. Asikainen S, Etemadzadeh H, Ainamo J. Reliability of standardised narrow strips in the periotron. $J$ Periodontol 1985; 56: 686-689.

17. Griffiths GS, Curtis MA, Wilton JMA. Selection of a filter 
paper with optimum properties for the collection of gingival crevicular fluid. $J$ Periodont Res 1988; 23: 33-38.

18. Leirskar J. In vitro experiments on gingival exudate measurements. II. The influence of different filter papers and ascending and descending chromatography on mobility. J Periodont Res 1971; 6: 23-27.

19. Hinrichs JE, Bandt CL, Smith JA, Golub LM. A comparision of 3 systems for quantifying gingival crevicular fluid with respect to linearity and the effects of qualitative differences in fluids. J Clin Periodontol 1984; 11: 652-661.

20. Lamster IB, Hartley LJ, Oshrain RL, Gordon JM. Evaluation and modification of spectrophotometric procedures for analysis of lactate dehydrogenase, betaglucuronidase and aryl sulphatase in human gingival crevicular fluid collected with filter-paper strips. Arch Oral Biol 1985; 30: 235-242.

21. Chapple ILC, Cross IA, Glenwright HD, Matthews JB.
Calibration and reliability of the Periotron 6000 for individual crevicular fluid samples. J Periodont Res 1995; 30: $73-79$.

22. Hinrichs JE, Bandt CL, Smith JA. Relative error (variability) associated with an improved instrument for measuring gingival crevicular fluid. J Periodontol 1984; 55: 294-298.

23. Nelkon M, Parker P. Capacitors. In: Advanced level physics, 4th edn, Ch 32. London: Heinemann Education Books Ltd, London, 1977: 677.

24. Sueda T, Cimasoni G, Held AJ. Histochemical study of human gingival fluid. Paradontologie 1966; 20: 141-147.

25. Ficara AJ, Levin MP, Grower MF, Kramer GD. A comparison of the glucose and protein content of gingival fluid from diabetics and non-diabetics. J Periodont Res 1975; 10: $171-175$.

26. Bang JS, Cimasoni G. Total protein in human crevicular fluid. J Dent Res 1975; 50: 638. 